\title{
SELECCIÓN DE MOTORES FUERA DE BORDA PARA EMBARCACIONES MENORES
}

\section{SELECCIÓN DE MOTORES FUERA DE BORDA PARA EMBARCACIONES MENORES}

\author{
Ing. Hugo Eliseo Gamarra Chinchay ${ }^{1}$, Ing. Anwar Julio Yarin Achachagua ${ }^{2}$, \\ Ing. Yasser Hipólito Yarin Achachagua ${ }^{3}$, Ing. Mierwen Palacios Aranda ${ }^{4}$
}

\begin{abstract}
RESUMEN
En la actualidad, el país requiere del uso de motores fuera de borda, debido a la gran cantidad de embarcaciones menores dedicadas a la actividad de recreo, pesca artesanal y guardacostas tanto para el uso lacustre, fluvial y marítimo. El estudio de las embarcaciones con el sistema de propulsión fuera de borda, varían entre la velocidad y la potencia, por lo cual el criterio desarrollado para la selección del motor y del hélice debe estar de acuerdo a los resultados del estudio hidrodinámicos del casco. En este proyecto damos la solución a los diferentes problemas de mantenimiento y reparación que puede ocurrir en el motor fuera de borda tomando en cuenta criterio de reducción de costos y desarrollo de un mantenimiento preventivo. Así mismo, comparamos ventajas y desventajas del clásico motor fuera de borda de 2 tiempos frente al moderno de 4 tiempos, desarrollando las diferencias en los sistemas de lubricación, refrigeración, arranque y otros. Importante también es ver un criterio de instalación y manejo del motor y distintos accesorios que la embarcación requiere. En conclusión en este proyecto nos proponemos obtener soluciones óptimas para los diferentes problemas de mantenimiento, etc.
\end{abstract}

Palabras clave.- Motores fuera de borda, Mantenimiento de motores.

\begin{abstract}
At the present time, the country requires of the use of motors outside hut, due to the great amount of shallow-draft vessels dedicated to the recreation activity, as much fishes artisan and coastguard vessel for the lacustrine, fluvial and marine use. The study of the boats with the system of propulsion outside hut, varies between the speed and the power, thus the criterion developed for the selection of the motor and the helix must be according to the hydrodynamic results of the study of the helmet. In this project we give to the solution to the different problems from maintenance and repair that can happen in the motor outside hut taking into account criterion of reduction of costs and development of a preventive maintenance. Also, we compared advantages and disadvantages of the classic motor outside hut of 2 times as opposed to modern of the 4 times, developing differences in the lubrication systems, refrigeration, starting and others. Important also it is to see a criterion of installation and handling of the motor and different accessories that the boat requires. In conclusion in this project we set out to obtain optimal solutions for the different problems from maintenance, etc.
\end{abstract}

Keywords.- Outboard motors, Engine maintenance.

\footnotetext{
${ }^{1}$ Magister Ing., docente de la Facultad de Ingeniería Mecánica de la Universidad Nacional de Ingeniería, ${ }^{2}$ Ing.Facultad de Ingeniería Mecánica de la Universidad Nacional de Ingeniería, ${ }^{3}$ Ing.Facultad de Ingeniería Mecánica de la Universidad Nacional de Ingeniería, ${ }^{4}$ Ing.Facultad de Ingeniería Mecánica de la Universidad Nacional de Ingeniería.
} 


\section{INTRODUCCION}

Los motores fuera de borda son de uso muy común en nuestro país. Y debido a que todos los trabajos de selección se hacen de manera empírica, el presente estudio pretende dar una herramienta útil y sencilla para poder adecuar la selección del motor a las características del casco sin dejar de lado el efecto de la interacción casco-hélice.

\section{DESARROLLO DEL TRABAJO}

Para poder realizar los objetivos primordiales de este proyecto se ha seguido el siguiente orden:
- Determinación de las líneas de forma de la embarcación.

- Estudio analítico de las líneas de forma usando el software de ingeniería MAXSURF.

- Selección de la potencia del motor basándose en los resultados anteriores.

- Selección de la hélice adecuada haciendo uso de formulaciones sobre propulsores.

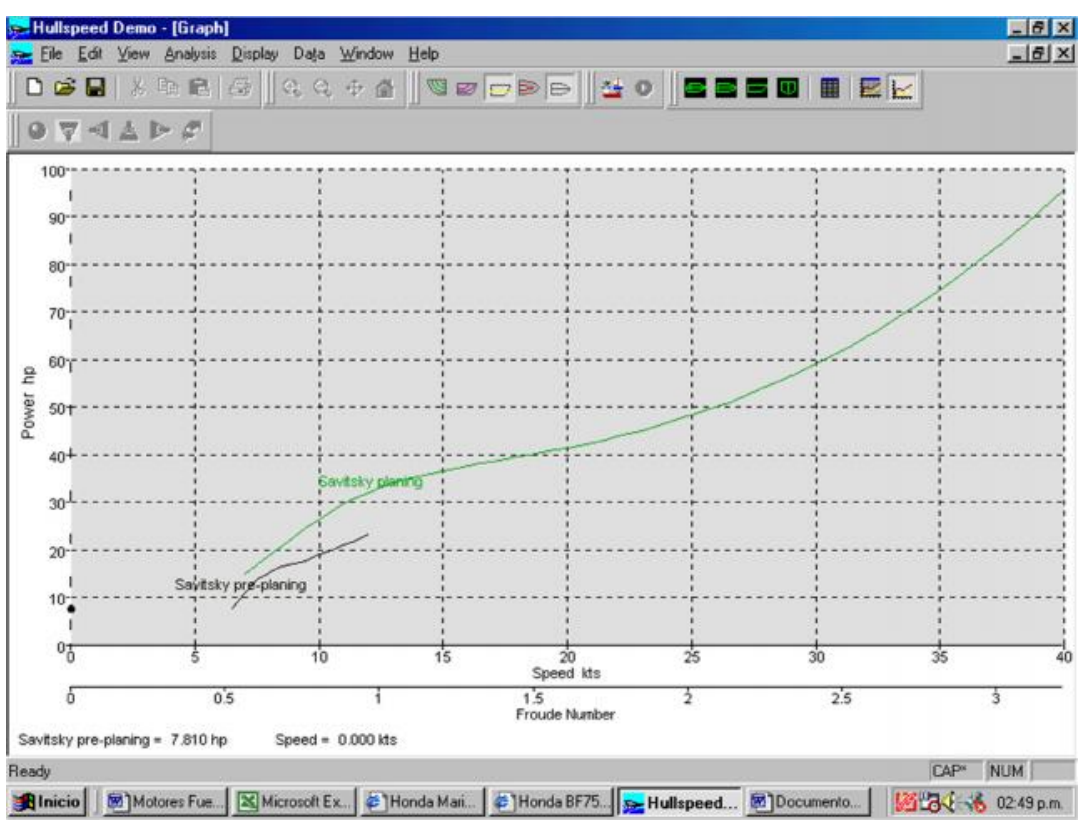

\section{RESULTADOS}

En este trabajo hemos hecho uso de las Series Sistemáticas de Savitsky para la determinación de la forma adecuada del casco de la embarcación y la potencia necesaria a la velocidad requerida. Para lo cual se empleo como herramienta el software de ingeniería MAXSURF. Obteniéndose los siguientes resultados:

La gráfica anterior muestra la relación Potencia (HP) vs Velocidad(kts). 


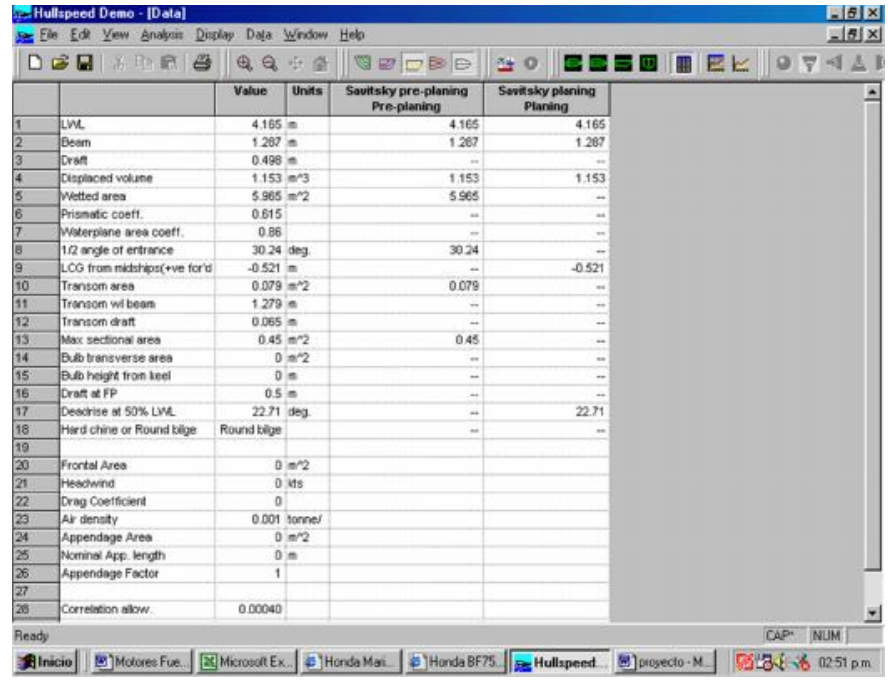

Esta tabla muestra los resultados obtenidos con el software MAXSURF, en el cual se observan los datos necesarios para el empleo de la Serie de
Savitsky. Ahora basándonos en la velocidad deseada para la embarcación seleccionamos el siguiente motor:

Tabla 1. Características de motor fuera de borda.

\begin{tabular}{|c|c|}
\hline Type & $\begin{array}{lll}\text { 4-Stroke } & \text { OHC } & 4 \\
\text { Cylinders } & & \\
\end{array}$ \\
\hline Displacement & $\begin{array}{l}1590 \text { cc } \\
\text { inches) }\end{array}$ \\
\hline Bore \& Stroke & $\begin{array}{l}75 \times 90 \mathrm{~mm}(3.00 \times 3.50 \\
\text { inches })\end{array}$ \\
\hline Full Throttle RPM & \\
\hline $\begin{array}{l}\text { Range } \\
\text { HP Rating @ }\end{array}$ & 5000-6000 RPM \\
\hline Propshaft & 75HP@5500 RPM \\
\hline Induction Scavenging & $\mathrm{SOHC}$ \\
\hline Valves per Cylinder & 3 \\
\hline Fuel Delivery & 4 Venturi \\
\hline Ignition System & Digital CDI \\
\hline Starting System & Electric \\
\hline Lubrication & Wet Sump \\
\hline Cooling System & Water Cooled \\
\hline Alternator & 16 Amps (202 Watts) \\
\hline Trim Range & $-4^{\circ}$ to $+12^{\circ}$ \\
\hline Tilt Range & $72^{\circ}$ \\
\hline
\end{tabular}

Una vez seleccionado el tipo de motor, la selección de la hélice adecuada se realizó en el siguiente orden:

Primera hélice.-

PROPELLER
$\begin{aligned} & \text { DIA. } X \text { PITCH_ } \\ & \text { NO. OF BLADES_ } \\ & \text { MFG. } \\ & \text { MATERIAL_ SUZUKI } \\ & \text { STAINLESS }\end{aligned}$




\begin{tabular}{|lr|}
\hline RPM & SPEED \\
1500 & 6.5 \\
2000 & 8.0 \\
2500 & 9.5 \\
3000 & 10.3 \\
3500 & 18.0 \\
4000 & 23.1 \\
4500 & 26.8 \\
5000 & 29.4 \\
5500 (w.o.T.) & 33.4 \\
\hline
\end{tabular}

Segunda hélice.-

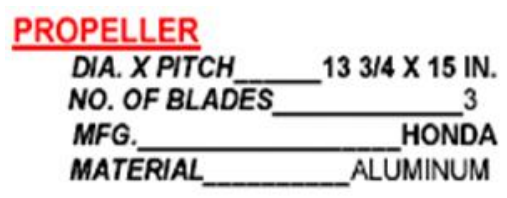

\begin{tabular}{|lr|}
\hline RPM & SPEED $(\mathrm{mph})$ \\
2500 & 8.51 \\
3000 & 12.49 \\
3500 & 16.23 \\
4000 & 20.28 \\
4500 & 24.72 \\
5000 & 27.59 \\
5500 (w.O.T.) & 30.36 \\
\hline
\end{tabular}

Luego se analizó el efecto de deslizamiento en la hélice y se obtuvieron los siguientes resultados:

\section{POTENCIA DEL MOTOR FUERA DE BORDA A 75HP-HONDA}

Tabla 2. Características requeridas.

\begin{tabular}{lllllll}
\hline $\begin{array}{l}\text { Reducción } \\
\text { Engranaje }\end{array}$ & $\begin{array}{c}\text { de Velocidad } \\
\text { 5000rpm }\end{array}$ & $\begin{array}{c}\text { a Velocidad a } \\
\text { 5500rpm }\end{array}$ & Diámetro & Paso & Velocidad 1 Velocidad 2 \\
\hline 2.33 & 29.4 & 33.4 & 13.75 & 16 & 5000 & 5500 \\
2.33 & 27.59 & 30.3 & 13.75 & 15 & 5000 & 5500 \\
\hline
\end{tabular}

Con los resultados anteriores se obtuvieron diferentes valores de deslizamiento:

Tabla 3. Valores de deslizamiento de la primera hélice.

\begin{tabular}{ll}
\hline \multicolumn{1}{c}{ velocidad rotacional } & \multicolumn{1}{c}{ velocidad rotacional } \\
\hline 57.00107296 & 62.70118026 \\
velocidad teórica 1 & velocidad teórica 2 \\
34.54610483 & 38.00071531 \\
deslizamiento 1 (\%) & deslizamiento 2 (\%) \\
\hline 14.89633882 & 12.10691765 \\
\hline
\end{tabular}

Tabla 4. Valores de deslizamiento de la segunda hélice.

\begin{tabular}{ll}
\hline $\begin{array}{l}\text { velocidad rotacional } \\
\mathbf{1}\end{array}$ & $\begin{array}{c}\text { velocidad rotacional } \\
\mathbf{2}\end{array}$ \\
\hline 57.00107296 & 62.70118026 \\
velocidad teórica 1 & velocidad teórica 2 \\
32.51398101 & 35.76537911 \\
deslizamiento 1 (\%) & deslizamiento 2(\%) \\
15.144196 & 15.2812 \\
\hline
\end{tabular}

Realizando diferentes comparaciones con los diferentes productos del mercado se obtuvieron los siguientes resultados: 


\section{POTENCIA DEL MOTOR FUERA DE BORDA A 75HP-YAMAHA}

Tabla 5. Características requeridas.

\begin{tabular}{|l|l|l|l|l|l|l|}
\hline $\begin{array}{l}\text { Reducción } \\
\text { de Engranaje }\end{array}$ & $\begin{array}{l}\text { Velocidad } \\
\text { a 5000rpm }\end{array}$ & $\begin{array}{l}\text { Velocidad } \\
\text { a 5500rpm }\end{array}$ & Diámetro & Paso & Velocidad 1 & Velocidad 2 \\
\hline 2.31 & 29.4 & 33.4 & 13.75 & 16 & 5000 & 5500 \\
\hline 2.31 & 27.59 & 30.3 & 13.75 & 15 & 5000 & 5500 \\
\hline
\end{tabular}

Tabla 6. Valores de deslizamiento de la primera hélice.

\begin{tabular}{ll}
\hline velocidad rotacional 1 & velocidad rotacional 2 \\
\hline 57.49458874 & 63.24404762 \\
velocidad teorica 1 & velocidad teorica 2 \\
34.8452053 & 38.32972583 \\
deslizamiento 1 (\%) & deslizamiento 2 $(\%)$ \\
15.62684235 & 12.86136471 \\
\hline
\end{tabular}

Tabla 7. Valores de deslizamiento de la segunda hélice.

\begin{tabular}{ll}
\hline velocidad rotacional 1 & velocidad rotacional 2 \\
\hline 57.49458874 & 63.24404762 \\
velocidad teórica 1 & velocidad teórica 2 \\
32.79548734 & 36.07503608 \\
deslizamiento 1 (\%) & deslizamiento 2 (\%) \\
15.872572 & 16.0084 \\
\hline
\end{tabular}

POTENCIA DEL MOTOR FUERA DE BORDA A 75HP-MERCURY

Tabla 8. Características requeridas.

\begin{tabular}{lllllll}
\hline $\begin{array}{l}\text { Reducción } \\
\text { de Engranaje }\end{array}$ & \multicolumn{2}{c}{ Velocidad Velocidad } & \multicolumn{4}{c}{ Velocidad Velocidad } \\
& 28.7 & 31.85 & 11.25 & 13 & 5000 & 5500 \\
2.07 & 31.4 & 34.8 & 11.5 & 14.255000 & 5500 \\
2.07 & & &
\end{tabular}

Tabla 9. Valores de deslizamiento de la primera hélice.

\begin{tabular}{ll}
\hline velocidad rotacional 1 & velocidad rotacional 2 \\
\hline 51.58011272 & 56.73812399 \\
velocidad teórica 1 & velocidad teórica 2 \\
32.02312985 & 35.22544283 \\
deslizamiento $1(\%)$ & deslizamiento 2 $(\%)$ \\
\multicolumn{1}{c}{10.37728} & 9.5824 \\
\hline
\end{tabular}


Tabla10. Valores de deslizamiento de la segunda hélice.

\begin{tabular}{ll}
\hline velocidad rotacional 1 & velocidad rotacional 2 \\
\hline 52.83816425 & 58.12198068 \\
velocidad teórica 1 & velocidad teórica 2 \\
34.88233787 & 38.37057166 \\
deslizamiento 1 $(\%)$ & deslizamiento 2 $(\%)$ \\
9.983097705 & 9.305495082 \\
\hline
\end{tabular}

\section{PROGRAMA DE INSPECCION Y MANTENIMIENTO DEL MOTOR DE 2 TIEMPOS}

Antes de cada uso:

1. Verifique que el interruptor de parada de emergencia pueda detener el motor.

2. Inspeccione visualmente el sistema de combustible para determinar si hay indicios de deterioro o fugas.

3. Verifique que el motor fuera de borda esté sujeto firmemente al espejo de popa.

4. Revise que no hayan componentes trabados ni sueltos en el sistema de dirección.

5. Inspeccione visualmente que los sujetadores de la varilla de la dirección estén debidamente ajustados.

6. Revise que las palas de la hélice no estén dañadas.

Después de cada uso:

1. Lave el sistema de enfriamiento del motor fuera de borda si se ha navegado en agua salada o contaminada.

2. Lave todos los depósitos de sal y la salida del escape de la hélice y caja de engranajes con agua dulce, si se ha navegado en agua salada.

Cada 100 horas de uso o una vez al año, según lo que ocurra:

1. Lubrique todos los puntos de lubricación. Lubrique con mayor frecuencia cuando se use en agua salada.

2. Inspeccione y limpie las bujías.

3. Examine el filtro del combustible del motor para ver si hay contaminantes.
4. Ajuste el (los) carburador(es) (si es necesario).

5. Examine el ajuste de puesta a punto del motor.

6. Examine los ánodos de control de corrosión. Examínelos con más frecuencia cuando se use en agua salada.

Cada 100 horas de uso o una vez al año, según lo que ocurra primero:

1. Drene y cambie el lubricante del cárter de engranajes.

2. Lubrique las estrías en el eje impulsor.

3. Examine el líquido del sistema de levante hidráulico.

4. Revise la batería.

5. Examine los ajustes del cable de control.

6. Quite los sedimentos del motor.

7. Examine que los pernos, tuercas y abrazaderas estén apretados.

Cada 300 Horas de uso o tres años:

1. Cambie el impulsor de la bomba de agua (más a menudo si ocurre un recalentamiento o si se nota una reducción en la presión del agua).

\section{PROGRAMA DE INSPECCION Y MANTENIMIENTO DEL MOTOR DE 4 TIEMPOS.}

Antes de cada uso:

1. Compruebe el nivel de aceite en el motor.

2. Verifique que el interruptor de parada de emergencia pueda detener el motor.

3. Inspeccione visualmente el sistema de combustible para determinar si hay indicios de deterioro o fugas. 
4. Verifique que el motor fuera de borda esté sujeto firmemente al espejo de popa.

5. Revise que no haya componentes trabados ni sueltos en el sistema de dirección.

6. Inspeccione visualmente que los sujetadores de la varilla de la dirección estén debidamente ajustados.

7. Revise que las palas de la hélice no estén dañadas.

Después de cada uso:

1. Lave el sistema de enfriamiento del motor fuera de borda si se ha navegado en agua salada o contaminada.

2. Lave todos los depósitos de sal y la salida del escape de la hélice y caja de engranajes con agua dulce, si se ha navegado en agua salada.

Cada 100 horas de uso o una vez al año, lo que ocurra primero:

1. Lubrique todos los puntos de lubricación. Lubrique con más frecuencia cuando se use en agua salada.

2. Cambie el aceite del motor y limpie el filtro del aceite.

3. El aceite debe cambiarse con más frecuencia cuando el motor funciona bajo condiciones adversas tales como el movimiento continuo del barco a velocidad baja para pescar.

4. Revise y limpie las bujías.

5. Inspeccione visualmente el termostato para ver si hay corrosión o si el resorte está roto. Cerciórese de que el termostato cierre por completo a la temperatura ambiente.

6. Examine el filtro del combustible del motor para ver si hay contaminantes.

7. Ajuste el carburador (si se requiere).

8. Examine el ajuste de puesta a punto del motor.

9. Examine los ánodos de control de corrosión. Examínelos con más frecuencia cuando se usen en agua salada.

10. Drene y cambie el lubricante de la caja de engranajes.

11. Lubrique las estrías del eje impulsor.

12. Revise y ajuste el espacio libre de la válvula, si es necesario.
13. Examine el líquido del sistema de levante hidráulico.

14. Revise la batería.

15. Examine los ajustes del cable de control.

16. Revise la correa de puesta a punto.

17. Quite los sedimentos del motor.

18. Compruebe que los pernos, tuercas y abrazaderas estén apretados.

Cada 300 horas de uso o tres años:

1. Cambie el impulsor de la bomba de agua (más a menudo si ocurre un recalentamiento o si se nota una reducción en la presión del agua).

\section{Características del motor de 4 tiempos}

Funcionamiento limpio y sin humos a cualquier velocidad, desde una velocidad de pesca hasta máxima aceleración.

El motor de 4 Tiempos ya cumple y excede los estándares de emisión EPA 2006.

La tecnología permite un funcionamiento suave y silencioso.

El diseño de la leva superior hace posible velocidades de ralentí a $750 \mathrm{rpm}$, haciendo del motor 4 Tiempos el motor ideal para practicar la pesca. Y no hace falta hacer la mezcla de combustible y aceite, ya que dos sistemas separados proporcionan la mezcla aire/combustible a los cilindros y el aceite a los puntos de lubricación.

\section{Características del motor de 2 tiempos}

Los motores son recios, fiables y fabricados para durar.

Ahorrará desde el primer momento, y seguirá beneficiándose con las ventajas de las excelentes relaciones peso/potencia que ofrece toda la gama.

Además, un menor peso facilita el transporte.

Fuera bordas de 2 y 4 Tiempos realizan las mismas cuatro fases: Admisión, compresión, explosión y escape. La diferencia fundamental reside en el número de carreras del pistón y las revoluciones del cigüeñal necesarias. 
Un motor de 4 Tiempos necesita cuatro carreras de pistón y dos revoluciones del cigüeñal para completar las cuatro fases, el doble que un motor de 2 Tiempos. Necesita unas válvulas de admisión y escape en la parte superior de cada cilindro que se abran y se cierren de manera independiente.

Los motores de 4 Tiempos no necesitan mezclar combustible y aceite, ya que cuentan con dos sistemas diferentes que suministran aire/combustible a los cilindros y aceite a todos los puntos de lubricación necesarios. Los motores de 4 Tiempos presentan las características de funcionamiento silencioso, suave, limpio y con economía de combustible inherentes a la tecnología de 4 Tiempos.

\section{CONCLUSIONES}

Logramos seleccionar el motor y hélice adecuada según el tipo de casco de la embarcación.

Logramos establecer un programa de mantenimiento preventivo en los motores fuera de borda.
Establecimos las principales ventajas y desventajas de los motores de 2 y 4 tiempos.

\section{REFERENCIAS}

1. V. Lewis E. "Principles of Naval Architecture Vol.II. 93-105. SNAME 601 Pavonia Avenue, Jersey City, N. J.

2. IBI-International Boat Industry, Reino Unido.

3. Menorquin Yachts "New Classic", España.

4. DVS-German Welding Society "Welding and Cutting, Alemania.

5. Diesel Progress International, Estados Unidos.

6. MarineLog, Estados Unidos.

7. BoatingLife, Estados Unidos.

8. Power Cruising, Estados Unidos.

9. Van Dam Nordia Yachts, Holanda.

10. Colegio de Ingenieros del Perú, "Mantenimiento Pruebas e Instalación de Motores Marinos Caterpillar", Lima-Perú.

Correspondencia: hegch16@yahoo.com.mx 